\title{
Computing Some Degree-Based Topological Indices of Honeycomb Networks
}

\author{
Lili Gu, ${ }^{1}$ Shamaila Yousaf, ${ }^{2,3}$ Akhlaq Ahmad Bhatti, ${ }^{2}$ Peng Xu $\mathbb{D}^{\mathbb{D}},{ }^{4}$ and Adnan Aslam $\mathbb{D}^{5}$ \\ ${ }^{1}$ Guangzhou Kangda Vocational Technical College, Guangzhou, Guangdong 510000, China \\ ${ }^{2}$ Department of Sciences and Humanities, National University of Computer and Emerging Sciences, Lahore Campus, B-Block, \\ Faisal Town, Lahore, Pakistan \\ ${ }^{3}$ Department of Mathematics, University of Gujrat, Hafiz Hayat Campus, Gujrat, Pakistan \\ ${ }^{4}$ Institute of Computing Science and Technology, Guangzhou University, Guangzhou 510006, China \\ ${ }^{5}$ Department of Natural Sciences and Humanities, University of Engineering and Technology, Lahore (RCET), Pakistan
}

Correspondence should be addressed to Adnan Aslam; adnanaslam15@yahoo.com

Received 16 August 2021; Accepted 30 November 2021; Published 4 January 2022

Academic Editor: Atila Bueno

Copyright (C) 2022 Lili Gu et al. This is an open access article distributed under the Creative Commons Attribution License, which permits unrestricted use, distribution, and reproduction in any medium, provided the original work is properly cited.

A topological index is a numeric quantity related with the chemical composition claiming to correlate the chemical structure with different chemical properties. Topological indices serve to predict physicochemical properties of chemical substance. Among different topological indices, degree-based topological indices would be helpful in investigating the anti-inflammatory activities of certain chemical networks. In the current study, we determine the neighborhood second Zagreb index and the first extended firstorder connectivity index for oxide network $\left(O X_{n}\right)$, silicate network $\left(S L_{n}\right)$, chain silicate network $\left(C S_{n}\right)$, and hexagonal network $\left(H X_{n}\right)$. Also, we determine the neighborhood second Zagreb index and the first extended first-order connectivity index for honeycomb network $\left(H C_{n}\right)$.

\section{Introduction}

Chemical graph theory/mathematical chemistry has made easier for the scientists to predict physicochemical properties of different chemical structures using topological indices. A numeric value assigned to a molecular graph (a graph portraying chemical compounds) is recognized as the topological index, and it remains unchanged under the isomorphism of the graph. For a given period, there is an increasing interest in topological indices that reflect some structural features of molecular compounds. Topological indices hold attention because they correlate well with certain chemical properties of molecular graphs. Also, they play an important role in the quantitative structure-activity relationship (QSAR) and quantitative structure property relationship (QSPR) studies [1]. To overview the structureactivity relationship, topological indices are required to effectively characterize structural features and bioactivity of chemical compounds [2-4]. In the current study, under discussion, molecular structures are nanostructures due to their design and applications.

All the graphs in present study are simple, undirected, finite, and connected. For a vertex $w \in V(G)$, we use the notation $N(w)$ for the set containing the vertices adjacent to $w$. The degree of a vertex $w$ is the cardinality of the set $N(w)$ and is denoted by $d_{w}$. Let $\mathcal{S}(w)$ denote the sum of degrees of the vertices adjacent to $w$. In other words, $\mathcal{S}(w)=\sum_{u w \in E(G)} d_{u}$ and $N(t)=\{v \in V(G) \mid t v \in E(G)\}$. For undefined terminologies related to graph theory, the author can read [5-7]. Consider the following general graph invariant:

$$
I(G)=\sum_{v w \in E(G)} f(\mathcal{S}(v), \mathcal{S}(w))
$$

Some special cases of the above invariants $I$ have already been appeared in mathematical chemistry. For example, if we take $f(\mathcal{S}(v), \mathcal{S}(w))=\mathcal{S}(v) \mathcal{S}(w)$ or $1 / \sqrt{\mathcal{S}(v) \mathcal{S}(w)}$, 
then $I$ gives neighborhood second Zagreb index $N M_{2}$ [8] and the first extended first-order connectivity index ${ }^{1} \chi_{1}$ $[9,10]$, respectively. These indices are defined as

$$
\begin{aligned}
{ }^{1} \chi_{1}(G) & =\sum_{v w \in E(G)} \frac{1}{\sqrt{S(v) S(w)}}, \\
N M_{2}(G) & =\sum_{v w \in E(G)} \mathcal{S}(v) \mathcal{S}(w) .
\end{aligned}
$$

Toropov et al. [11] proved that extended molecular connectivity indices have good correlation with the boiling point of chemical structures. Detail about theoretical and computation aspects of some families of chemical structures can be viewed in [12-16].

In this report, we are interested in five well-known chemical structures, name as oxide network $\left(O X_{n}\right)$, honeycomb network $\left(H C_{n}\right)$, silicate network $\left(S L_{n}\right)$, chain silicate network $\left(C S_{n}\right)$, and hexagonal network $\left(H X_{n}\right)$. As far as authors are aware, neighborhood second Zagreb index and the first extended first-order connectivity index of oxide network $\left(O X_{n}\right)$, silicate network $\left(S L_{n}\right)$, chain silicate network $\left(C S_{n}\right)$, hexagonal network $\left(H X_{n}\right)$, and honeycomb network $\left(H C_{n}\right)$ have not been investigated until now, and therefore, the study under consideration is an addition in the aforesaid direction.

\section{Motivation}

Since topological indices are useful to predict the physicochemical properties of chemical compounds, therefore, it is important to analyze the correlation of the topological indices investigated. Yousaf et al. [17] proved that the correlation of entropy and eccentric factor of octane isomers with neighborhood second Zagreb index and the first extended first-order connectivity index has high accuracy. In addition, topological indices are moreover used for discrimination against octane isomers. The discrimination ability of an index has remarkable application within the coding and the computer processing of molecular structures (isomers).

Denote by $x_{q, r}(H)$ (or $x_{q, r}$, when terms are not confusing) the number of edges in $H$ with end-vertex degrees $q$ and $r$. Two graphs $H_{1}$ and $H_{2}$ are called edge-equivalent graphs if they are satisfying $x_{q, r}\left(H_{1}\right)=x_{q, r}\left(H_{2}\right)$ for all $q$ and $r$ with $1 \leq q \leq r \leq \Delta$. A topological index $T I$ is called edgeequivalent topological index if it satisfies $T I\left(H_{1}\right)=T I\left(H_{2}\right)$ for every pair of edge-equivalent graphs $H_{1}$ and $H_{2}$. We notice that the bond incident degree indices $[18,19]$ (BID indices for short [20]) are edge-equivalent topological indices. General form of the BID indices is

$$
\operatorname{BID}(H)=\sum_{v w \in E(G)} g\left(d_{v}, d_{w}\right)=\sum_{q \leq r} x_{q, r} g(q, r) .
$$

where $g(q, r)$ is a bivariate symmetric function. It is worth noting that most of the degree-based topological indices used in mathematical chemistry are BID indices. These indices may be produced from equation (4) based on the selection of $g(q, r)$.
To study the discriminatory performance of the topological index $N M_{2}$ and ${ }^{1} \chi_{1}$, the class of octane isomers are considered which are representing the 8 -vertex trees. These 18 molecular graphs of octane isomers are shown in Figure 1. Figure 1 makes it easy to see that trees $\mathrm{O}_{3}$ and $\mathrm{O}_{4}$ are edgeequivalent, but their corresponding first extended first-order connectivity indices and neighborhood second Zagreb indices are not the same: ${ }^{1} \chi_{1}\left(O_{3}\right)=0.097,{ }^{1} \chi_{1}\left(O_{4}\right)=0.096$, $N M_{2}\left(O_{3}\right)=106$, and $N M_{2}\left(O_{4}\right)=107$. Similarly, the trees $\mathrm{O}_{11}$ and $\mathrm{O}_{12}$ are edge-equivalent graphs possessing different first extended first-order connectivity indices and neighborhood second Zagreb indices: ${ }^{1} \chi_{1}\left(O_{11}\right)=0.086$, ${ }^{1} \chi_{1}\left(O_{12}\right)=0.087, N M_{2}\left(O_{13}\right)=136$, and $N M_{2}\left(O_{4}\right)=137$.

Thus, the topological index $N M_{2}$ and ${ }^{1} \chi_{1}$ does not belong to the class of edge-equivalent topological indices. Subsequently, we can presume that the topological indices $N M_{2}$ and ${ }^{1} \chi_{1}$ are identified by a better discriminatory power than the conventional BID indices. Particularly, Wang et al. [21] proved that all the molecular graphs of 18 octane isomers have different $N M_{2}$ and ${ }^{1} \chi_{1}$ values. It is worth mentioning that, in certain specific cases, the discriminatory capacity of $\mathrm{NM}_{2}$ and ${ }^{1} \chi_{1}$ is limited, but still better than that of the BID indices. This can be explained by examining the corresponding edgeequivalent graphs illustrated in Figure 2: we have $N M_{2}\left(D_{1}\right)=$ 451 and ${ }^{1} \chi_{1}\left(D_{1}\right)=1.09$, but $N M_{2}\left(D_{2}\right)=N M_{2}\left(D_{3}\right)=448$ and ${ }^{1} \chi_{1}\left(D_{2}\right)={ }^{1} \chi_{1}\left(D_{3}\right)=1.08$ (of course, these three graphs are identical for any arbitrary BID index; in other words, with the help of an arbitrary BID index, it is difficult to distinguish between the graphs $D_{1}, D_{2}$, and $D_{3}$ ).

\section{Main Results and Discussion}

Multiprocessor interconnection networks are frequently needed to join many identical processor-memory pairs that are repeated, each one is referred to as a processing node. Message passing rather than shared memory is frequently used for synchronization of a communication between the processing nodes for the execution of the program. Multiprocessor interconnection networks are most attracted networks due to the accessibility of powerful and inexpensive microprocessors and memory chips. By repeating regular polygons, periodic plane tessellations can be easily built. For direct interconnection networks, this design is very important as it offers high global performance. Some parallel networks that originate from popular meshes are silicate, chain silicate, hexagonal, honeycomb, and oxide networks. Such networks have very attractive topological properties that have been investigated in various ways in [22-33].

3.1. Oxide Networks. Oxide networks are important in investigating silicate networks. Oxide networks are obtained by the deletion of silicon nodes from a silicate network (see Figure 3 ). An oxide network of dimension $n$ is denoted as $O X_{n}$. The order and size of $O X_{n}$ is $9 n^{2}+3 n$ and $18 n^{2}$, respectively. In oxide networks $O X_{n}$, the edge set can be partitioned into six sets depending on degree summation of neighbors of end vertices. This partition is presented in Table 1. 


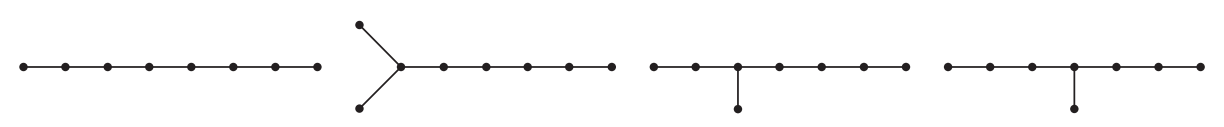

(a)

(b)

(c)

(d)

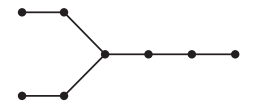

(e)

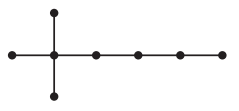

(f)

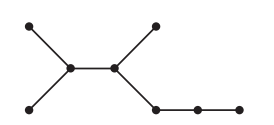

(g)

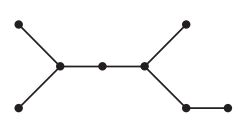

(h)

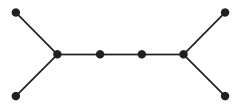

(i)

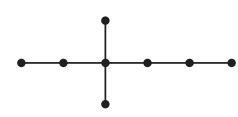

(j)

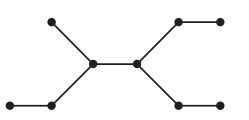

(k)

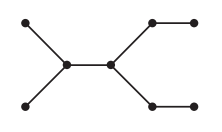

(1)

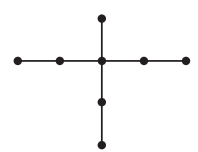

(m)

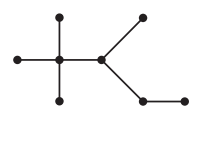

(n)

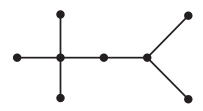

(o)

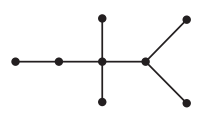

(p)

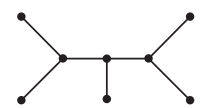

(q)

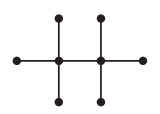

(r)

Figure 1: Graphs of octane isomers. (a) $O_{1}$. (b) $O_{2}$. (c) $O_{3}$. (d) $O_{4}$. (e) $O_{5}$. (f) $O_{6}$. (g) $O_{7}$. (h) $O_{8}$. (i) $O_{9}$. (j) $O_{10}$. (k) $O_{11}$. (l) $O_{12}$. (m) $O_{13}$. (n) $O_{14}$. (o) $O_{15} \cdot\left(\right.$ p) $O_{16} \cdot\left(\right.$ q) $O_{17} \cdot\left(\right.$ r) $O_{18}$.

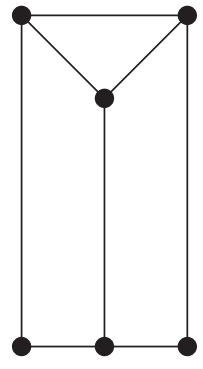

(a)

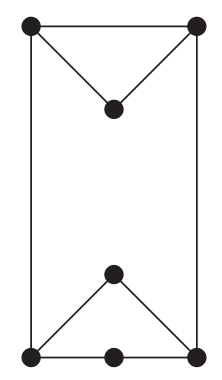

(b)

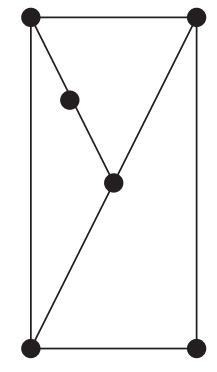

(c)

Figure 2: Edge-equivalent graphs. (a) $D_{1}$. (b) $D_{2}$. (c) $D_{3}$.

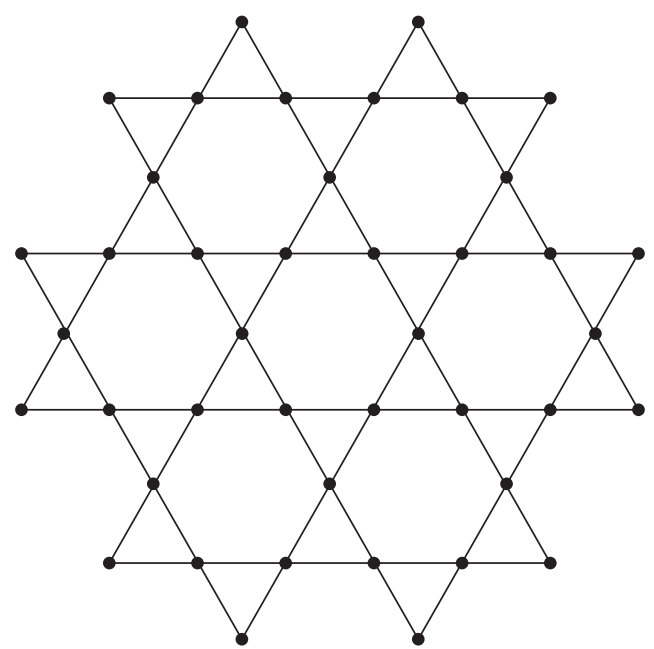

Figure 3: An oxide network $\mathrm{OX}_{2}$. 
TABLE 1: Edge partitions of $O X_{n}$.

\begin{tabular}{lc}
\hline$(S(v) S(w))$, where $v w \in E\left(O X_{n}\right)$ & Cardinality \\
\hline$(8,12)$ & 12 \\
$(12,14)$ & 12 \\
$(16,16)$ & $18 n^{2}-36 n+18$ \\
$(8,1)$ & $12(n-1)$ \\
$(14,16)$ & $12(n-1)$ \\
$(14,14)$ & $6(2 n-3)$ \\
\hline
\end{tabular}

In the following theorems, we investigate ${ }^{1} \chi_{1}$ and $N M_{2}$ of $O X_{n}$

Theorem 1. The ${ }^{1} \chi_{1}$ index of oxide networks $O X_{n}$ is given by

$$
\begin{aligned}
{ }^{1} \chi_{1}\left(O X_{n}\right)= & \frac{9}{8} n^{2}+\left(\frac{3}{\sqrt{7}}+\frac{3}{\sqrt{14}}-\frac{39}{28}\right) n \\
& +\frac{3}{\sqrt{6}}-\frac{3}{\sqrt{7}}+\frac{6}{\sqrt{42}}-\frac{3}{\sqrt{14}}-\frac{9}{56} .
\end{aligned}
$$

Proof. We use equation (2) and Table 1 to calculate the expression for ${ }^{1} \chi_{1}$ index of the oxide networks $O X_{n}$ :

$$
\begin{aligned}
{ }^{1} \chi_{1}\left(O X_{n}\right)= & \sum_{v w \in E(G)} \frac{1}{\sqrt{\mathcal{S}(v) \mathcal{S}(w)}} \\
= & (12) \frac{1}{\sqrt{8 \times 12}}+(12) \frac{1}{\sqrt{12 \times 14}} \\
& +12(n-1) \frac{1}{\sqrt{8 \times 14}}+6(2 n-3) \frac{1}{\sqrt{14 \times 14}} \\
& +12(n-1) \frac{1}{\sqrt{14 \times 16}} \\
& +\left(18 n^{2}-36 n+18\right) \frac{1}{\sqrt{16 \times 16}} \\
= & \frac{9}{8} n^{2}+\left(\frac{3}{\sqrt{7}}+\frac{3}{\sqrt{14}}-\frac{39}{28}\right) n \\
& +\frac{3}{\sqrt{6}}-\frac{3}{\sqrt{7}}+\frac{6}{\sqrt{42}}-\frac{3}{\sqrt{14}}-\frac{9}{56} .
\end{aligned}
$$

$$
\begin{aligned}
{ }^{1} \chi_{1}\left(H C_{n}\right) & =\sum_{v w \in E(G)} \frac{1}{\mathcal{S}(v) \mathcal{S}(w)} \\
& =(6) \frac{1}{\sqrt{5 \times 5}}+12(n-1) \frac{1}{\sqrt{5 \times 7}}+6(n-1) \frac{1}{\sqrt{7 \times 9}}+\left(9 n^{2}-21 n+12\right) \frac{1}{\sqrt{9 \times 9}} \\
& =n^{2}+\left(\frac{2}{\sqrt{7}}+\frac{12}{\sqrt{35}}-\frac{7}{3}\right) n+\frac{38}{15}-\frac{12}{\sqrt{35}}-\frac{2}{\sqrt{7}} .
\end{aligned}
$$

Theorem 2. The $\mathrm{NM}_{2}$ index of oxide networks $\mathrm{OX}$ is given by

$$
N M_{2}\left(O X_{n}\right)=4608 n^{2}-2832 n+216
$$

Proof. To calculate the expression for neighborhood second Zagreb index of oxide networks $O X_{n}$, we use equation (3) and Table 1 to get the required result:

$$
\begin{aligned}
N M_{2}\left(O X_{n}\right)= & \sum_{v w \in E(G)} \mathcal{S}(v) \mathcal{S}(w) \\
= & (12)(8 \times 12)+(12)(12 \times 14) \\
& +6(2 n-3)(14 \times 14)+12(n-1)(8 \times 14) \\
& +\left(18 n^{2}-36 n+18\right)(16 \times 16) \\
& +12(n-1)(14 \times 16) \\
= & 4608 n^{2}-2832 n+216 .
\end{aligned}
$$

3.2. Honeycomb Networks. In material sciences, honeycomb networks have vital role in different fields especially in computer graphics, cellular phone base stations, and image processing and in representing benzenoid hydrocarbons. Honeycomb networks are shaped by the iterative use of hexagonal tiles in a specific design. We denote the $n$-dimensional honeycomb network with $n$ hexagons between central and boundary hexagon by $H C_{n} . H C_{n}$ is constructed by adding layer of hexagon around $H C_{n-1}$ (see Figure 4 ). The order and size of $H C_{n}$ is $6 n^{2}$ and $9 n^{2}-3 n$, respectively. Table 2 shows partition of edges.

By using partition of edges of $H C_{n}$, we investigate ${ }^{1} \chi_{1}$ and $\mathrm{NM}_{2}$ of $\mathrm{HC}_{n}$ in the following theorems.

Theorem 3. The ${ }^{1} \chi_{1}$ index of Honeycomb network $H C_{n}$ is given by

${ }^{1} \chi_{1}\left(H C_{n}\right)=n^{2}+\left(\frac{2}{\sqrt{7}}+\frac{12}{\sqrt{35}}-\frac{7}{3}\right) n+\frac{38}{15}-\frac{12}{\sqrt{35}}-\frac{2}{\sqrt{7}}$.

Proof. We use equation (2) and Table 2 to calculate the expression for ${ }^{1} \chi_{1}$ index of the Honeycomb network $H C_{n}$ : 


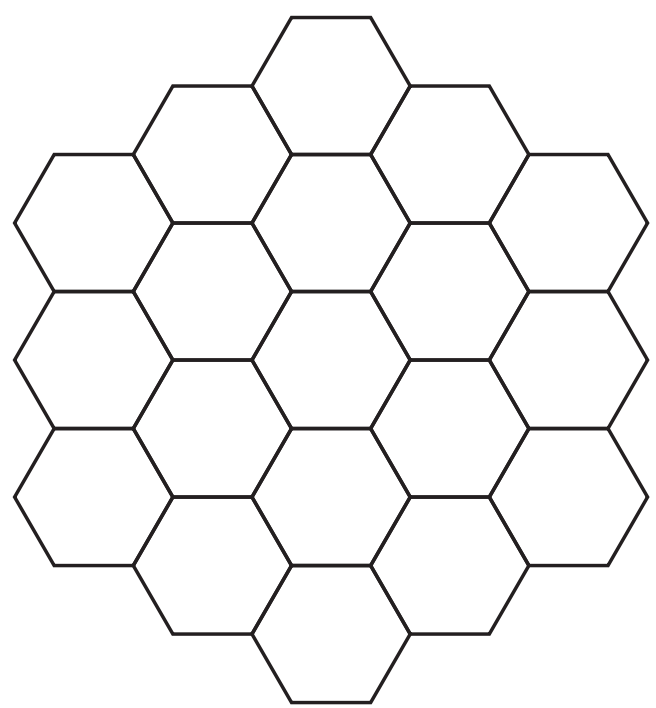

FIgURe 4: Honeycomb network.

TABle 2: Edge partitions of $H C_{n}$.

\begin{tabular}{lc}
\hline$(S(v) S(w))$, where $v w \in E\left(H C_{n}\right)$ & Cardinality \\
\hline$(5,5)$ & 6 \\
$(5,7)$ & $12(n-1)$ \\
$(7,9)$ & $6(n-1)$ \\
$(9,9)$ & $9 n^{2}-21 n+12$ \\
\hline
\end{tabular}

Theorem 4. The $\mathrm{NM}_{2}$ index of Honeycomb network $\mathrm{HC}_{n}$ is given by

$$
N M_{2}\left(H C_{n}\right)=729 n^{2}-903 n+324 .
$$

Proof. To calculate the expression for $N M_{2}$ index of Honeycomb network $H C_{n}$, we use equation (3) and Table 2, to get the required result:

$$
\begin{aligned}
N M_{2}\left(H C_{n}\right)= & \sum_{v w \in E(G)} \mathcal{S}(v) \mathcal{S}(w) \\
= & (6)(5 \times 5)+12(n-1)(5 \times 7) \\
& +6(n-1)(7 \times 9)+\left(9 n^{2}-21 n+12\right)(9 \times 9) \\
= & 729 n^{2}+903 n+324 .
\end{aligned}
$$

3.3. Silicate Networks. Silicates are constituent elements of the common minerals that form the rock and, absolutely, the most important and the most complicated minerals. The elementary unit of silicates' minerals are tetrahedron $\mathrm{SiO}_{4}$. Silicate is produced by melting metal oxides or metal sand carbonates. $\mathrm{SiO}_{4}$ tetrahedral content is nearly found in all silicate materials. A silicate sheet is formed by different types of sequences of tetrahedrons which are connected by adjoining oxygen vertices to other tetrahedrons in a plane. Cyclic silicates are networks that result in rings of different lengths after being bound by sharing oxygen vertices. Figures 5 and 6 present certain cyclic and sheet silicates, respectively. Other types of silicates are also available and are illustrated in Figure 7. From a chemical perspective, the oxygen atoms are in fact the corner vertices of tetrahedron $\mathrm{SiO}_{4}$ and silicon atom is its central vertex. Graphically, we describe the central atom as silicon vertex, corner atoms as oxygen vertices, and edges are bonds between them. Figure 8 illustrates a tetrahedron of $\mathrm{SiO}_{4}$. We denote a silicate array with $n$ hexagons between center and border of silicate sheet by $S L_{n}$. Figure 9 illustrates a three-dimensional silicate network. The order and size of $S L_{n}$ is $15 n^{2}+3 n$ and $36 n^{2}$, respectively. In the following theorems, the silicate networks are analyzed through certain graph invariants.

In the next theorem, we computed ${ }^{1} \chi_{1}$ and $N M_{2}$ of the $S L_{n}$ in the following way.

Theorem 5. The ${ }^{1} \chi_{1}$ index of silicate networks is given by

$$
\begin{aligned}
{ }^{1} \chi_{1}\left(S L_{n}\right)= & \left(\frac{3}{\sqrt{15}}+\frac{3}{5}\right) n^{2}+\left(\frac{8}{3 \sqrt{5}}+\frac{4}{3 \sqrt{6}}-\frac{5}{\sqrt{15}}+\frac{4}{3 \sqrt{10}}-\frac{16}{45}\right) n \\
& +\frac{4}{\sqrt{10}}-\frac{8}{3 \sqrt{5}}-\frac{4}{3 \sqrt{6}}+\frac{2}{\sqrt{15}}+\frac{2}{3 \sqrt{2}}-\frac{4}{3 \sqrt{10}}-\frac{1}{15} .
\end{aligned}
$$



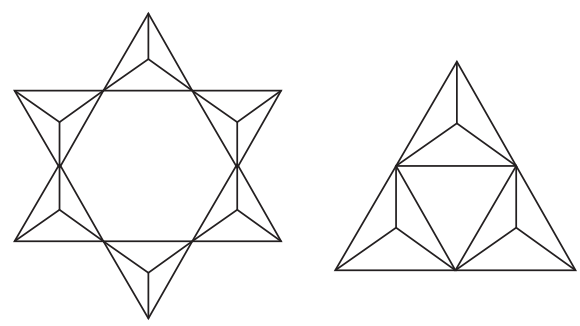

Figure 5: Cyclic silicates.

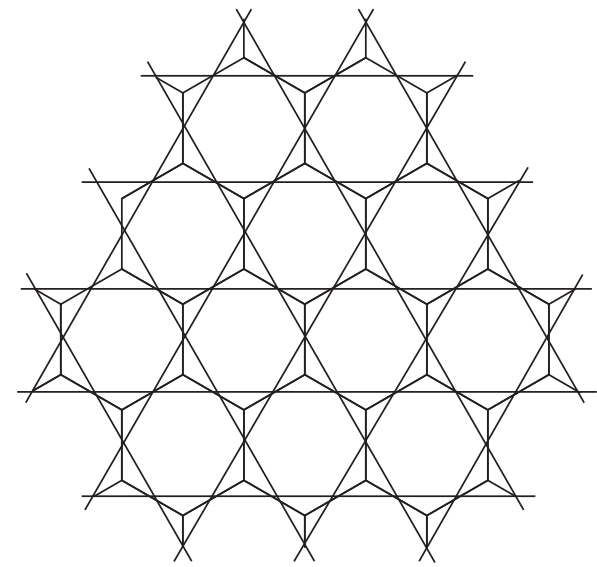

FIGURE 6: Sheet silicates.

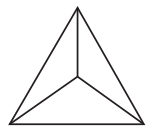

(a)

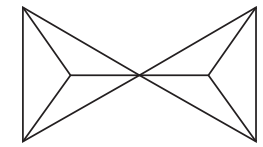

(b)

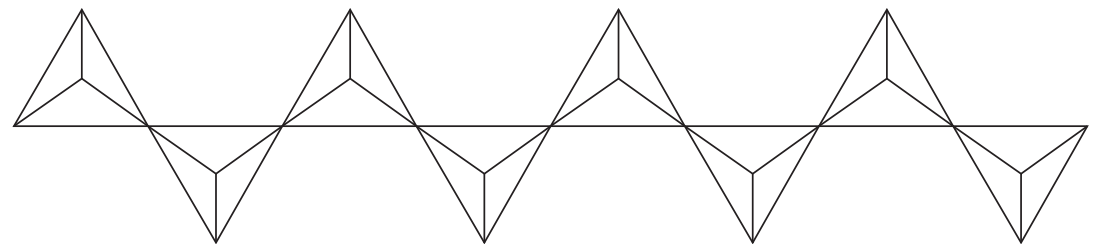

(c)

FIgUre 7: (a) Ortho, (b) pyro, and (c) chain silicates.

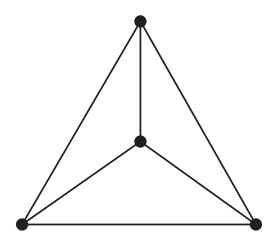

Figure 8: $\mathrm{SiO}_{4}$ tetrahedron. 


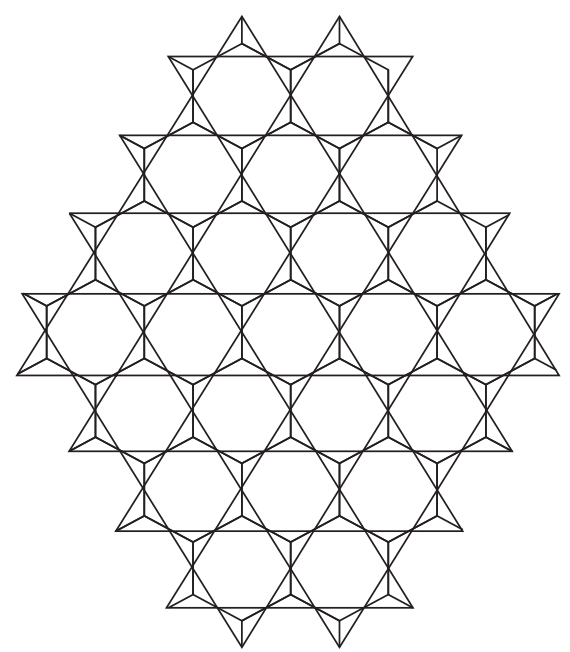

Figure 9: For $n=3$, silicate network $S L_{n}$.

Proof. We use equation (2) and Table 3 to calculate the expression for ${ }^{1} \chi_{1}$ index of the silicate networks:

$$
\begin{aligned}
& { }^{1} \chi_{1}\left(S L_{n}\right)=\sum_{v w \in E(G)} \frac{1}{\sqrt{\mathcal{S}(v) \mathcal{S}(w)}} \\
& =(6 n) \frac{1}{\sqrt{15 \times 15}}+(24) \frac{1}{\sqrt{15 \times 24}}+24(n-1) \frac{1}{\sqrt{15 \times 27}}+12(n-1) \frac{1}{\sqrt{18 \times 27}} \\
& +\left(18 n^{2}-30 n+12\right) \frac{1}{\sqrt{18 \times 30}}+(12) \frac{1}{\sqrt{24 \times 27}}+6(2 n-3) \frac{1}{\sqrt{27 \times 27}} \\
& +12(n-1) \frac{1}{\sqrt{27 \times 30}}+\left(18 n^{2}-36 n+18\right) \frac{1}{\sqrt{30 \times 30}} \\
& =\frac{2 n}{5}+\frac{4}{\sqrt{10}}+\frac{8(n-1)}{3 \sqrt{5}}+\frac{4(n-1)}{3 \sqrt{6}}+\frac{6 n^{2}-10 n+4}{2 \sqrt{15}}+\frac{2}{3 \sqrt{2}}+\frac{2(2 n-3)}{9} \\
& +\frac{4(n-1)}{3 \sqrt{10}}+\frac{6 n^{2}-12 n+6}{10} \\
& =\left(\frac{3}{\sqrt{15}}+\frac{3}{5}\right) n^{2}+\left(\frac{8}{3 \sqrt{5}}+\frac{4}{3 \sqrt{6}}-\frac{5}{\sqrt{15}}+\frac{4}{3 \sqrt{10}}-\frac{16}{45}\right) n \\
& +\frac{4}{\sqrt{10}}-\frac{8}{3 \sqrt{5}}-\frac{4}{3 \sqrt{6}}+\frac{2}{\sqrt{15}}+\frac{2}{3 \sqrt{2}}-\frac{4}{3 \sqrt{10}}-\frac{1}{15} . \\
& N M_{2}\left(S L_{n}\right)=25920 n^{2}-13230 n+702 \text {. } \\
& N M_{2}\left(S L_{n}\right)=\sum_{v w \in E(G)} \mathcal{S}(v) \mathcal{S}(w) \\
& =(6 n)(15 \times 15)+12(n-1)(18 \times 27) \\
& +(24)(15 \times 24) \\
& +12(n-1)(27 \times 30)+24(n-1)(15 \times 27) \\
& +\left(18 n^{2}-30 n+12\right)(18 \times 30)
\end{aligned}
$$

Theorem 6. The $\mathrm{NM}_{2}$ index of silicate networks is given by

Proof. To calculate the expression for $\mathrm{NM}_{2}$ index of silicate networks, we use equation (3) and Table 3 to get the required result: 
TABLE 3: Edge partition of $L S_{n}$.

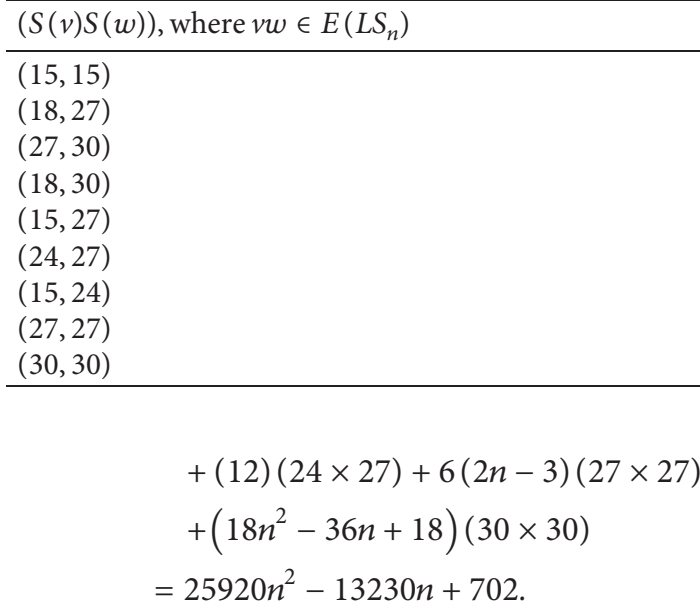

3.4. Chain Silicate Networks. Chain silicate is acquired by linear arrangement of tetrahedron. $n$-dimensional chain silicate networks are characterized as follows. A network of $n$-dimensional chain silicate stands for $C S_{n}$ is acquired by linear arrangement of $n$ tetrahedron. The order and size of $C S_{n}$ with $n>1$ is $3 n+1$ and and $6 n$, respectively. A network of $n$-dimensional chain silicate is illustrated in Figure 10.

Cardinality
$6 n$
$12(n-1)$
$12(n-1)$
$18 n^{2}-30 n+12$
$24(n-1)$
12
24
$6(2 n-3)$
$18 n^{2}-36 n+18$

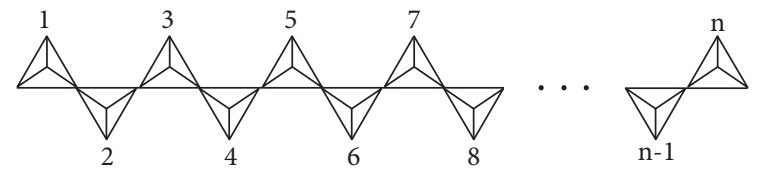

FIGURE 10: $n$-dimensional chain silicate network.

Tables 4 and 5 suggest such a partition that we use to compute certain topological indices. Now, within the following theorems, we analyzed the chain silicate networks $C S_{n}$ via topological indices.

$$
\begin{aligned}
{ }^{1} \chi_{1}\left(C S_{3}\right) & =\sum_{v w \in E(G)} \frac{1}{\sqrt{\mathcal{S}(v) \mathcal{S}(w)}} \\
& =(6) \frac{1}{\sqrt{12 \times 12}}+(1) \frac{1}{\sqrt{15 \times 15}}+(6) \frac{1}{\sqrt{12 \times 21}}+(1) \frac{1}{\sqrt{21 \times 21}}+(4) \frac{1}{\sqrt{15 \times 21}} \\
& =\frac{1}{2}+\frac{1}{15}+\frac{1}{\sqrt{7}}+\frac{1}{21}+\frac{4}{\sqrt{315}} .
\end{aligned}
$$

The following results provide the investigation of certain topological indices of $C S_{n}$.

Theorem 7. The ${ }^{1} \chi_{1}$ index of $C S_{n}$ is given by

$$
{ }^{1} \chi_{1}\left(C S_{n}\right)= \begin{cases}\frac{1}{2}+\frac{1}{\sqrt{6}} & \text { if } n=2 \\ \frac{1}{2}+\frac{1}{15}+\frac{1}{\sqrt{7}}+\frac{1}{21}+\frac{4}{\sqrt{315}} & \text { if } n=3 \\ \left(\frac{13}{120}+\frac{2}{3 \sqrt{10}}\right) n+\frac{1}{5}+\frac{1}{\sqrt{7}}-\frac{2}{\sqrt{10}}+\frac{4}{\sqrt{315}}+\frac{1}{\sqrt{126}} & \text { if } n>3 .\end{cases}
$$


TABle 4: Edge partition of $C S_{n}$.

\begin{tabular}{lc}
\hline$(S(v) S(w))$, where $v w \in E\left(C S_{n}\right)$ & Cardinality \\
\hline$(12,12)$ & 6 \\
$(12,21)$ & 6 \\
$(15,15)$ & $n-2$ \\
$(15,21)$ & 4 \\
$(15,24)$ & $4 n-12$ \\
$(21,24)$ & 2 \\
$(24,24)$ & $n-4$ \\
\hline
\end{tabular}

TABle 5: Edge partition of $\mathrm{CS}_{3}$.

\begin{tabular}{lc}
\hline$(S(v) S(w))$, where $v w \in E\left(C S_{3}\right)$ & Cardinality \\
\hline$(12,12)$ & 6 \\
$(15,15)$ & 1 \\
$(12,21)$ & 6 \\
$(15,21)$ & 4 \\
$(21,21)$ & 1 \\
\hline
\end{tabular}

Proof. There are three cases that need to be examined in order to prove these outcomes. First, we demonstrate this result for $n=2$. In the graph $G=C S_{2}$, the edge partition is of the type $(\mathcal{S}(12), \mathcal{S}(12))$ and $(\mathcal{S}(12), \mathcal{S}(18))$ and each of this partition has six edges. Then,

$$
\begin{aligned}
{ }^{1} \chi_{1}\left(C S_{2}\right) & =\sum_{v w \in E(G)} \frac{1}{\sqrt{\mathcal{S}(v) \mathcal{S}(w)}} \\
& =(6) \frac{1}{\sqrt{12 \times 12}}+(6) \frac{1}{\sqrt{12 \times 18}} \\
& =\frac{1}{2}+\frac{1}{\sqrt{6}} .
\end{aligned}
$$

For the case $n=3$, the edge partition $C S_{n}$ is presented in Table 5. Now, ${ }^{1} \chi_{1}\left(C S_{3}\right)$ can be computed as follows:

Let us now turn to the third case of $n>3$. To calculate the expression for ${ }^{1} \chi_{1}$ index of the chain silicate networks $C S_{n}$, we use equation (2) and Table 4 to obtain the required result:

$$
\begin{aligned}
{ }^{1} \chi_{1}\left(C S_{3}\right)= & \sum_{v w \in E(G)} \frac{1}{\sqrt{\mathcal{S}(v) \mathcal{S}(w)}} \\
= & (6) \frac{1}{\sqrt{12 \times 12}}+(1) \frac{1}{\sqrt{15 \times 15}}+(6) \frac{1}{\sqrt{12 \times 21}}+(1) \frac{1}{\sqrt{21 \times 21}}+(4) \frac{1}{\sqrt{15 \times 21}} \\
& +4(n-3) \frac{1}{\sqrt{15 \times 24}}+(2) \frac{1}{\sqrt{21 \times 24}}+(n-4) \frac{1}{\sqrt{24 \times 24}} \\
= & \left(\frac{13}{120}+\frac{2}{3 \sqrt{10}}\right) n+\frac{1}{5}+\frac{1}{\sqrt{7}}-\frac{2}{\sqrt{10}}+\frac{4}{\sqrt{315}}+\frac{1}{\sqrt{126}} .
\end{aligned}
$$

Theorem 8. The $\mathrm{NM}_{2}$ index of the chain silicate networks $C S_{n}$ is given by

$$
N M_{2}\left(C S_{n}\right)= \begin{cases}2160 & \text { if } n=2, \\ 4302 & \text { if } n=3 \\ 2241 n-2430 & \text { if } n>3\end{cases}
$$

Proof. There are three cases that need to be examined in order to prove these outcomes. First, we demonstrate this result for $n=2$. In the graph $G=C S_{2}$, the edge partition is of the type $(\mathcal{S}(12), \mathcal{S}(12))$ and $(\mathcal{S}(12), \mathcal{S}(18))$ and each of this partition has six edges. Then,

$$
\begin{aligned}
N M_{2}\left(C S_{2}\right) & =\sum_{v w \in E(G)} \mathcal{S}(v) \mathcal{S}(w) \\
& =(6)(12 \times 12)+(6)(12 \times 18) \\
& =2160 .
\end{aligned}
$$

$$
\begin{aligned}
N M_{2}\left(C S_{3}\right)= & \sum_{v w \in E(G)} \mathcal{S}(v) \mathcal{S}(w) \\
= & (6)(12 \times 12)+(1)(15 \times 15)+(6)(12 \times 21) \\
& +(1)(21 \times 21)+(4)(15 \times 21) \\
= & 4302 .
\end{aligned}
$$

Let us now turn to the third case of $n>3$. To calculate the expression for the $N M_{2}$ index of the chain silicate networks $C S_{n}$, we use equation (3) and Table 4 to obtain the required result:

$$
\begin{aligned}
N M_{2}\left(C S_{n}\right)= & \sum_{v w \in E(G)} \mathcal{S}(v) \mathcal{S}(w) \\
= & (6)(12 \times 12)+(6)(12 \times 21)+(4)(15 \times 21) \\
& +(n-2)(15 \times 15) \\
& +4(n-3)(15 \times 24)+(n-4)(24 \times 24)+(2)(21 \times 24) \\
= & 2241 n-2430 .
\end{aligned}
$$

For the case $n=3$, the edge partition $C S_{n}$ is presented in Table 5. Now, $\mathrm{NM}_{2}\left(\mathrm{CS}_{3}\right)$ can be computed as 
3.5. Hexagonal Networks. It is a known fact that tilling a plane by regular polygon can be done only with regular triangles, hexagons, and squares. A hexagonal network can be obtained by using triangles (see Figure 11). A hexagonal network with $n$ vertices in each side of the hexagon is denoted by $H X_{n}$. Let $n>1$; then, order and size of $H X_{n}$ is $3 n^{2}-3 n+1$ and $9 n^{2}-15 n+6$, respectively. Figure 11 depicts the graph of $\mathrm{HX}_{6}$. Tables 6-8 depict a partition that we use to compute the topological indices of hexagonal networks $H X_{n}$ for $n \geq 3$. Now, we investigate aforementioned indices of hexagonal networks $H X_{n}$.

In the following theorems, we investigate ${ }^{1} \chi_{1}$ and $N M_{2}$ of $H X_{n}$.

Theorem 9. The ${ }^{1} \chi_{1}$ index of hexagonal networks $H X_{n}$ is given by

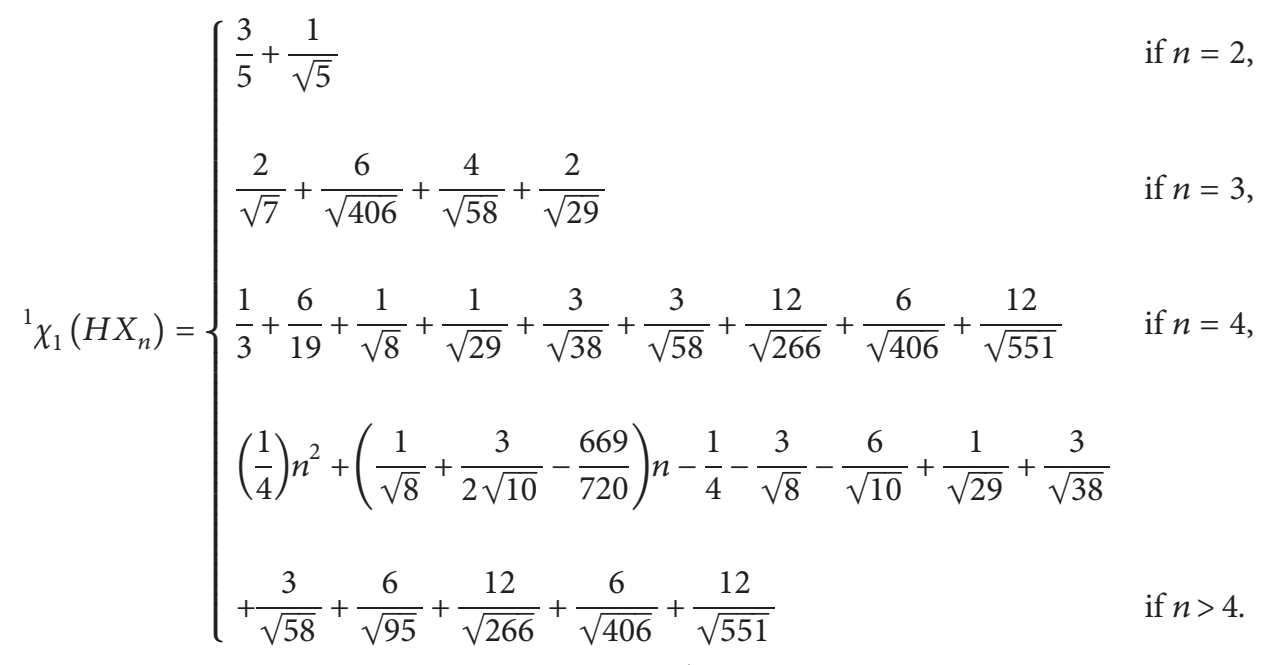

Proof. There are four cases that need to be examined in order to prove these outcomes. First, we demonstrate this result for $n=2$. In the graph $G=H X_{2}$, the edge partition is of the type $(\mathcal{S}(10), \mathcal{S}(10))$ and $(\mathcal{S}(10), \mathcal{S}(18))$ and each of this partition has six edges. Then,

$$
\begin{aligned}
{ }^{1} \chi_{1}\left(H X_{2}\right) & =\sum_{v w \in E(G)} \frac{1}{\sqrt{\mathcal{S}(v) \mathcal{S}(w)}} \\
& =(6) \frac{1}{\sqrt{10 \times 10}}+(6) \frac{1}{\sqrt{10 \times 18}} \\
& =\frac{3}{5}+\frac{1}{\sqrt{5}} .
\end{aligned}
$$

For the case $n=3$, the edge partition $\mathrm{HX}_{3}$ is presented in Table 6. Now, ${ }^{1} \chi_{1}\left(H X_{3}\right)$ can be computed as

$$
\begin{aligned}
{ }^{1} \chi_{1}\left(H X_{3}\right)= & \sum_{v w \in E(G)} \frac{1}{\sqrt{\mathcal{S}(v) \mathcal{S}(w)}} \\
= & (12) \frac{1}{\sqrt{14 \times 18}}+(6) \frac{1}{\sqrt{14 \times 29}}+(12) \frac{1}{\sqrt{18 \times 29}} \\
& +(12) \frac{1}{\sqrt{29 \times 36}} \\
= & \frac{2}{\sqrt{7}}+\frac{6}{\sqrt{406}}+\frac{4}{\sqrt{58}}+\frac{2}{\sqrt{29}} .
\end{aligned}
$$

For the case $n=4$, the edge partition $\mathrm{HX}_{4}$ is presented in Table 7. Now, ${ }^{1} \chi_{1}\left(H X_{4}\right)$ can be computed as

$$
{ }^{1} \chi_{1}\left(H X_{4}\right)=\sum_{v w \in E(G)} \frac{1}{\sqrt{\mathcal{S}(v) \mathcal{S}(w)}}
$$

$$
\begin{aligned}
= & (12) \frac{1}{\sqrt{14 \times 19}}+(6) \frac{1}{\sqrt{19 \times 19}}+(6) \frac{1}{\sqrt{14 \times 29}} \\
& +(12) \frac{1}{\sqrt{19 \times 29}} \\
& +(12) \frac{1}{\sqrt{19 \times 32}}+(12) \frac{1}{\sqrt{29 \times 32}}+(6) \frac{1}{\sqrt{29 \times 36}} \\
& +(12) \frac{1}{\sqrt{32 \times 36}}+(12) \frac{1}{\sqrt{36 \times 36}} \\
= & \frac{1}{3}+\frac{6}{19}+\frac{1}{\sqrt{8}}+\frac{1}{\sqrt{29}}+\frac{3}{\sqrt{38}}+\frac{3}{\sqrt{58}}+\frac{12}{\sqrt{266}} \\
& +\frac{6}{\sqrt{406}}+\frac{12}{\sqrt{551}} .
\end{aligned}
$$




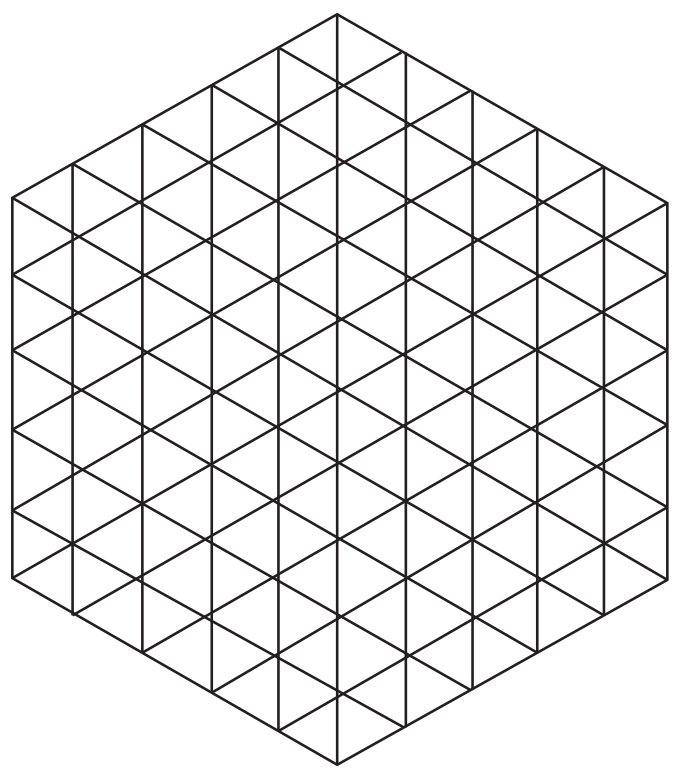

Figure 11: For $n=6$, hexagonal network $H X_{n}$.
TABLE 6: Edge partitions of $H X_{3}$.

\begin{tabular}{lc}
\hline$(S(v) S(w))$, where $v w \in E\left(H X_{3}\right)$ & Cardinality \\
\hline$(14,18)$ & 12 \\
$(14,29)$ & 6 \\
$(18,29)$ & 12 \\
$(29,36)$ & 12 \\
\hline
\end{tabular}

TABle 7: Edge partitions of $H X_{4}$.

\begin{tabular}{lc}
\hline$(S(v) S(w))$, where $v w \in E\left(H X_{4}\right)$ & Cardinality \\
\hline$(14,19)$ & 12 \\
$(19,19)$ & 6 \\
$(14,29)$ & 6 \\
$(19,29)$ & 12 \\
$(19,32)$ & 12 \\
$(29,32)$ & 12 \\
$(29,36)$ & 6 \\
$(32,36)$ & 12 \\
$(36,36)$ & 12 \\
\hline
\end{tabular}

Let us now turn to the case of $n>4$. To calculate the expression for ${ }^{1} \chi_{1}$ index of hexagonal networks $H X_{n}$, we use equation (2) and Table 8 to obtain the required result:

$$
\begin{aligned}
{ }^{1} \chi_{1}\left(H X_{n}\right)= & \sum_{v w \in E(G)} \frac{1}{\sqrt{\mathcal{S}(v) \mathcal{S}(w)}} \\
= & (12) \frac{1}{\sqrt{14 \times 19}}+(12) \frac{1}{\sqrt{19 \times 20}}+6(n-5) \frac{1}{\sqrt{20 \times 20}}+(12) \frac{1}{\sqrt{19 \times 29}} \\
& +(12) \frac{1}{\sqrt{19 \times 32}}+12(n-4) \frac{1}{\sqrt{20 \times 32}}+(6) \frac{1}{\sqrt{14 \times 29}}+(12) \frac{1}{\sqrt{29 \times 32}} \\
& +(6) \frac{1}{\sqrt{29 \times 36}}+12(n-3) \frac{1}{\sqrt{32 \times 36}}+6(n-4) \frac{1}{\sqrt{32 \times 32}} \\
& +\left(9 n^{2}-51 n+72\right) \frac{1}{\sqrt{36 \times 36}} \\
= & \left(\frac{1}{4}\right) n^{2}+\left(\frac{1}{\sqrt{8}}+\frac{3}{2 \sqrt{10}}-\frac{669}{720}\right) n-\frac{1}{4}-\frac{3}{\sqrt{8}}-\frac{6}{\sqrt{10}}+\frac{1}{\sqrt{29}}+\frac{3}{\sqrt{38}} \\
& +\frac{3}{\sqrt{58}}+\frac{6}{\sqrt{95}}+\frac{12}{\sqrt{266}}+\frac{6}{\sqrt{406}}+\frac{12}{\sqrt{551}} .
\end{aligned}
$$

Theorem 10. The $\mathrm{NM}_{2}$ index of the hexagonal networks $H X_{n}$ is given by

$$
N M_{2}\left(H X_{n}\right)= \begin{cases}1680, & \text { if } n=2, \\ 24252, & \text { if } n=3, \\ 68478, & \text { if } n=4, \\ 11664 n^{2}-36048 n+26040, & \text { if } n>4 .\end{cases}
$$

Proof. There are four cases that need to be examined in order to prove these outcomes. First, we demonstrate this result for $n=2$. In the graph $G=H X_{2}$, the edge partition is of the type $(\mathcal{S}(10), \mathcal{S}(10))$ and $(\mathcal{S}(10), \mathcal{S}(18))$ and each of this partition has six edges. Then,

For the case $n=3$, the edge partition $H X_{n}$ is presented in Table 6. Now, $N M_{2}\left(H X_{3}\right)$ can be computed as 
TABLE 8: Edge partitions of $H X_{n}$.

\begin{tabular}{lc}
\hline$(S(v) S(w))$, where $v w \in E\left(H X_{n}\right)$ & Cardinality \\
\hline$(14,19)$ & 12 \\
$(19,20)$ & 12 \\
$(20,20)$ & $6(n-5)$ \\
$(14,29)$ & 6 \\
$(19,29)$ & 12 \\
$(19,32)$ & 12 \\
$(20,32)$ & $12(n-4)$ \\
$(29,32)$ & 12 \\
$(29,36)$ & 6 \\
$(32,32)$ & $6(n-4)$ \\
$(32,36)$ & $12(n-3)$ \\
$(36,36)$ & $9 n^{2}-51 n+72$ \\
\hline
\end{tabular}

$$
\begin{aligned}
N M_{2}\left(H X_{3}\right)= & \sum_{v w \in E(G)} \frac{1}{\sqrt{\mathcal{S}(v) \mathcal{S}(w)}} \\
= & (12)(14 \times 18)+(6)(14 \times 29) \\
& +(12)(18 \times 29)+(12)(29 \times 36) \\
= & 24252 .
\end{aligned}
$$

For the case $n=4$, the edge partition $H X_{n}$ is presented in Table 7. Now, $\mathrm{NM}_{2}\left(H X_{3}\right)$ can be computed as

$$
\begin{aligned}
N M_{2}\left(H X_{4}\right)= & \sum_{v w \in E(G)} \frac{1}{\sqrt{\mathcal{S}(v) \mathcal{S}(w)}} \\
= & (12)(14 \times 19)+(6)(19 \times 19)+(6)(14 \times 29) \\
& +(12)(19 \times 29) \\
& +(12)(19 \times 32)+(12)(29 \times 32)+(6)(29 \times 36) \\
& +(12)(32 \times 36)+(12)(36 \times 36) \\
= & 68478 .
\end{aligned}
$$

Let us now turn to the third case of $n>4$. To calculate the expression for $\mathrm{NM}_{2}$ index of hexagonal networks $H X_{n}$, we use equation (3) and Table 8 to obtain the required result:

$$
\begin{aligned}
N M_{2}\left(H X_{n}\right)= & \sum_{v w \in E(G)} \frac{1}{\sqrt{\mathcal{S}(v) \mathcal{S}(w)}} \\
= & (12)(14 \times 19)+(12)(19 \times 20)+6(n-5)(20 \times 20)+(12)(19 \times 29) \\
& +(12)(19 \times 32)+12(n-4)(20 \times 32)+(6)(14 \times 29)+(12)(29 \times 32) \\
& +(6)(29 \times 36)+12(n-3)(32 \times 36)+6(n-4)(32 \times 32) \\
& +\left(9 n^{2}-51 n+72\right)(36 \times 36) \\
= & 11664 n^{2}-36048 n+26040 .
\end{aligned}
$$

\section{Conclusion}

The aim of this paper is to discuss the degree-based topological indices of several nanotube networks. In this paper, we determined the neighborhood second Zagreb index and the first extended first-order connectivity index for oxide network $\left(O X_{n}\right)$, silicate network $\left(S L_{n}\right)$, chain silicate network $\left(C S_{n}\right)$, and hexagonal network $\left(H X_{n}\right)$. Also, we determined the neighborhood second Zagreb index and the first extended first-order connectivity index for honeycomb network $\left(H C_{n}\right)$. In this paper, results achieved demonstrate the promising opportunities to apply for chemical sciences. It is very helpful to know more about nanotube networks that have become the central interest of fundamental sciences and study their topological indices that will be completely useful to understand their underlying topologies.

\section{Data Availability}

No data were used to support the findings of the study.

\section{Conflicts of Interest}

On behalf of all authors, the corresponding author states that there are no conflicts of interest.

\section{Acknowledgments}

This work was funded in part by the National Natural Science Foundation of China (Grant no. 62002079).

\section{References}

[1] M. Randić, "Novel graph theoretical approach to heteroatoms in quantitative structure-activity relationship," Chemometrics and Intelligent Laboratory Systems, vol. 10, pp. 213-227, 1991.

[2] A. T. Balaban, I. Motoc, D. Bonchev, O. Mekenyan, M. Charton, and I. Motoc, "Topological indices for structure activity relationship. In steric effort in drug design," Topics in Current Chemistry, vol. 114, pp. 21-55, 1983. 
[3] A. T. Balaban, "Topological indices and their uses: a new approach for the coding of alkanes," Journal of Molecular Structure: THEOCHEM, vol. 165, no. 3-4, pp. 243-253, 1988.

[4] A. T. Balaban, "Chemical graphs," Theoretica Chimica Acta, vol. 53, no. 4, pp. 355-375, 1979.

[5] B. Bollobás, Modern Graph Theory, Springer-Verlag, Berlin, Germany, 1998.

[6] J. L. Gross, J. Yellen, Graph Theory, CRC Press, Boca Raton, Florida, 2000.

[7] N. Trinajsti, Chemical Graph Theory, CRC Press, Boca Raton, Florida, 1992.

[8] T. Réti, A. Ali, P. Varga, and E. Bitay, "Some properties of the neighborhood first Zagreb index," Discrete Math. Lett, vol. 2, pp. 10-17, 2019.

[9] D. Bonchev and L. B. Kier, "Topological atomic indices and the electronic charges in alkanes," Journal of Mathematical Chemistry, vol. 9, no. 1, pp. 75-85, 1992.

[10] B. Zhou and N. Trinajstić, "On extended connectivity indices," Journal of Mathematical Chemistry, vol. 46, no. 4, pp. 1172-1180, 2009.

[11] A. A. Toropov, A. P. Toropova, T. T. Ismailov, N. L. Voropaeva, and I. N. Ruban, "Extended molecular connectivity: p," Journal of Structural Chemistry, vol. 38, no. 6, pp. 965-969, 1997.

[12] A. Aslam, Y. Bashir, S. Ahmad, and W. Gao, "On topological indices of certain dendrimer structures," Zeitschrift für Naturforschung A, vol. 72, no. 6, pp. 559-566, 2017.

[13] A. Aslam, S. Ahmad, and W. Gao, "On certain topological indices of boron triangular nanotubes," Zeitschrift für Naturforschung A, vol. 72, no. 8, pp. 711-716, 2017.

[14] W. Gao, Z. Iqbal, M. Ishaq, R. Sarfraz, M. Aamir, and A. Aslam, "On eccentricity-based topological indices study of a class of pcdendrimers," Biomolecules, vol. 8, no. 3, p. 71, 2018.

[15] Z. Iqbal, M. Ishaq, A. Aslam, and W. Gao, "On eccentricity based topological descriptors of water soluble dendrimers," Zeitschrift für Naturforschung C, vol. 74, no. 1-2, 2018.

[16] A. Aslam, S. Ahmad, M. A. Binyamin, and W. Gao, "Calculating topological indices of certain OTIS interconnection networks," Open Chemistry, vol. 17, no. 1, pp. 220-228, 2019.

[17] S. Yousaf, A. A. Bhatti, and A. Aslam, "Study of carbon nanbotubes and boron nanotubes using degree based topological indices," Polycyclic Aromatic Compounds, pp. 1-14, 2021.

[18] D. Vukičević and J. Durdević, "Bond additive modeling 4. QSPR and QSQR studies of the variable adriatic indices," Croatica Chemica Acta, vol. 84, pp. 87-91, 2011.

[19] D. Vukičević and J. Đurđević, "Bond additive modeling 10. Upper and lower bounds of bond incident degree indices of catacondensed fluoranthenes," Chemical Physics Letters, vol. 515, no. 1-3, pp. 186-189, 2011.

[20] A. Ali, Z. Raza, and A. A. Bhatti, "Bond incident degree (BID) indices of polyomino chains: a unified approach," Applied Mathematics and Computation, vol. 287-288, pp. 28-37, 2016.

[21] Y. Wang, S. Yousaf, A. Ahmad Bhatti, and A. Aslam, "Analyzing the expressions for nanostructures via topological indices," Arabian Journal of Chemistry, vol. 15, no. 1, Article ID 103469, 2022.

[22] F. García, J. Solano, I. Stojmenovic, and M. Stojmenovic, "Higher dimensional hexagonal networks," Journal of Parallel and Distributed Computing, vol. 63, pp. 1164-1172, 2003.

[23] S. Hayat and M. Imran, "Computation of topological indices of certain networks," Applied Mathematics and Computation, vol. 240, pp. 213-228, 2014.
[24] S. Hayat, M. Imran, and M. Yasir, "On topological indices of certain interconnection networks," Applied Mathematics and Computation, vol. 244, pp. 936-951, 2014.

[25] M. N. Husin, A. Ariffin, and M. Alaeiyan, "The neighbourhood polynomial of certain networks," Journal of Information and Optimization Sciences, vol. 41, no. 4, pp. 941-948, 2020.

[26] M. N. Husin, R. Hasni, and M. Imran, "More results on computation of topological indices of certain networks," International Journal of Networking and Virtual Organisations, vol. 17, no. 1, pp. 46-63, 2017.

[27] E. V. Konstantinova, "The discrimination ability of some topological and information distance indices for graphs of unbranched hexagonal systems," Journal of Chemical Information and Computer Sciences, vol. 36, no. 1, pp. 54-57, 1996.

[28] J.-B. Liu, M. K. Shafiq, H. Ali, A. Naseem, N. Maryam, and S. S. Asghar, "Topological indices of $\mathrm{mth}$ chain silicate graphs," Mathematics, vol. 7, no. 1, p. 42, 2019.

[29] F. Garcia Nocetti, I. Stojmenovic, and J. Jingyuan Zhang, "Addressing and routing in hexagonal networks with applications for tracking mobile users and connection rerouting in cellular networks," IEEE Transactions on Parallel and Distributed Systems, vol. 13, no. 9, pp. 963-971, 2002.

[30] B. Rajan, A. William, C. Grigorious, and S. Stephen, "On certain topological indices of silicate, honeycomb and hexagonal networks," J. Comp. Math. Sci, vol. 5, pp. 530-535, 2012.

[31] Z. Raza and M. Essa, "M-Polynomial and degree based topological indices of some nanostructures," Symmetry, vol. 12, no. 5, p. 831, 2020.

[32] Z. Raza, "Leap Zagreb connection numbers for some networks models," Indonesian Journal of Chemistry, vol. 20, no. 6, p. 1407, Article ID 1407, 2020.

[33] I. Stojmenovic, "Honeycomb networks: topological properties and communication algorithms," IEEE Transactions on Parallel and Distributed Systems, vol. 8, no. 10, pp. 1036-1042, 1997. 